\title{
Efectos del déficit nutricional en la creatividad de los alumnos de educación primaria
}

\author{
María Luisa Matalinares Calvet \\ Universidad Nacional Mayor de San Marcos
}

En la presente investigación, de carácter sustantivo, se empleó el método descriptivo, con un diseño causal comparativo. Se propuso estudiar los efectos del déficit nutricional crónico en la creatividad de los niños que cursan educación primaria. El trabajo se realizó con dos grupos apareados de 644 alumnos, con y sin déficit nutricional, a quienes se evaluó en fluidez, flexibilidad, originalidad y organización. Los resultados revelaron que no existen diferencias entre los niños con déficit nutricional crónico y el grupo de contraste, excepto en dos casos: cuarto y sexto grados, y únicamente en los indicadores de originalidad y organización. Los niños adecuadamente nutridos obtuvieron medias significativamente superiores a las de los niños con déficit nutricional crónico.

Déficit nutricional / educación primaria / fluidez / flexibilidad / originalidad / creatividad

\section{Effects of nutritional deficit in the creativity of students of primary school}

It is a substantive work in which a descriptive method with a comparative causal design was applied. The purpose was to study chronic nutritional deficit in the creativity of children who attend primary school. It was performed with two matched groups of 644 students with and without nutritional deficit, who were evaluated in fluidity, flexibility, originality and organization. The results showed that no differences existed between children with chronic nutritional deficit and the contrast group, except in two cases: fourth and sixth grade students; and only in the indicators of originality and organization. Children suitably nourished obtained means significantly superior to children with chronic nutritional deficit.

nutritional deficit / primary education / fluidity / flexibility / originality / creativity

Correo electrónico: maria_luisa93@hotmail.com 
El déficit nutricional es uno de los problemas de mayor incidencia en el mundo. Napoli y Horton (1997) señalan que el déficit nutricional sigue siendo una de las primeras causas de mortalidad y morbilidad en los países en desarrollo. Se ha calculado que más de 400 millones de personas en todo el mundo presentan déficit nutricional $\mathrm{y}$ se espera que este número aumente a medida que lo hace la superpoblación. En el informe del Unicef de 1997 se señala que mueren más de 12 millones de niños en todo el planeta, en gran parte por causa de este problema. De los 150 países reportados por Unicef en su informe sobre "Estado mundial de la infancia" en 1997, el Perú ocupa el lugar 65. Según la tasa de mortalidad en menores de 5 años, se estima en 42 por mil nacidos vivos. Según el Banco Mundial, esta cifra coloca al Perú "entre los peores de América Latina y el Caribe" (Iguíñiz, 2004).

El informe estadístico de la infancia 2000 señala que, a escala mundial, existen 840 millones de personas que sufren desnutrición crónica; además, que en los últimos 20 años $1.200 \mathrm{mi}$ llones, es decir una de cada cinco personas, incluidos unos 600 millones de niños, viven en la pobreza. En América Latina casi el $40 \%$ de las familias vive en extrema pobreza crítica, aproximadamente 60 millones de niños pertenecen a esas familias y el $20 \%$ de estas vive en un estado de pobreza absoluta. Actualmente en América Latina más del 50\% de los niños menores de 6 años presentan algún grado de desnutrición. Ahora el $41 \%$ de estos niños sufren de retardo de crecimiento (Sfeir y Aguayo, 2000).

Ahora bien, en el Perú, como en el resto del mundo, se desarrolla una serie de investigaciones tendientes a determinar los efectos del déficit nutricional, tanto en el desarrollo orgánico como psíquico del niño, y el cúmulo de investigaciones realizadas hasta aquí constituyen interesantes aportes al conocimiento del tema.

No obstante, si bien hay acuerdo entre los distintos investigadores en torno a los efectos del déficit nutricional en el desarrollo orgánico (Webb, 1973; Oski, 1974; Chun, 1971; Popkim \& Lim Ibáñez, 1982; Gratham \& Mc.Gregor, 1982; citados por Pollit, 1984; Gratham \& Mc Gregor, 1995; citado por Pollit, 2002; Kallin, 1973; Merril \& Felson, 1976, entre otros); no ocurre así en lo que se refiere a sus efectos en el desarrollo psíquico (Matalinares, 1989; Yarlequé, 1989; Ocrospoma, Yarlequé, Matalinares \& Fernández Baca, 1991). Tales desacuerdos revelan, de un lado, las dificultades metodológicas para estudiar la influencia del déficit nutricional en el desarrollo psíquico, distinguiendo adecuadamente los efectos que en la variable dependiente tienen los factores ambientales, vinculados estrechamente con aquella. De otro lado, tiene relación con la necesidad de profundizar las investigaciones, a fin de 
distinguir la influencia del déficit nutricional en cada uno de los diferentes procesos psíquicos superiores, que dadas sus peculiaridades (leyes particulares del desarrollo, estructuras cerebrales y procesos bioquímicos involucrados) podrían no ser afectados en la misma dimensión.

Es importante señalar que una gran cantidad de estudios realizados emplea sujetos en edad preescolar. En los casos en que se toman niños en edad escolar se refieren, en gran parte, al rendimiento en la escuela, que si bien es un indicador de la calidad de los procesos psíquicos del niño, no lo es absolutamente; pues en este influyen una serie de factores que deben tenerse en cuenta para una adecuada interpretación de los resultados. En este sentido se han estudiado los efectos del déficit nutricional en el CI (Cravioto \& otros, 1965; Mockeberg, 1973; Winick, Meyer \& Harris, 1975; Stoch \& Smithe, 1976; Bartel \& otros, 1977; Das \& Soysa, 1978; Horweg \& Stanfield, 1979; Galler \& otros, 1983; Pollit \& Granoff, 1974; entre otros). Pero se ha estudiado también en otras variables cognitivas, como la memoria, la atención, la percepción e inclusive en el desarrollo motriz (Champakan \& otros, 1968; Nwuga, 1977; Matalinares, 1989; Yarlequé, 1989; Zavala, 1991, \& Ocrospoma, Yarlequé Matalinares \& Fernández Baca, 1991). Pero muy poco se ha trabajado sobre el pensamiento y la creatividad.
De allí que hacer estudios en fenómenos como la creatividad tiene mucha importancia, más aun si autores como Merril y Felson (1976) ponen énfasis en los efectos nocivos del déficit nutricional sobre el aprendizaje, indicando que los procesos cognitivos suelen ser muy afectados.

Conocer los efectos del déficit nutricional en la creatividad es de particular importancia, no solo para la educación escolarizada, en la cual las alteraciones y déficit psicológicos suelen tener consecuencias muy negativas en el rendimiento del educando (Torres, 1996); también las tiene en el desenvolvimiento personal del individuo dentro de la sociedad, pues tal déficit, de acuerdo con su magnitud, puede llegar a limitar el desarrollo intelectual de las personas, colocándole en una situación desventajosa frente al medio, afectando con ello su autoestima y limitando el potencial humano de la sociedad de que se trate.

Hay que tomar en cuenta que el enorme desarrollo tecnológico alcanzado en los últimos años, sobre todo en el campo de la cibernética y las telecomunicaciones, han generado nuevas condiciones de vida en el marco de un mundo globalizado. Estas condiciones se caracterizan -entre otras cosas- por un cambio radical en la educación que deben tener los hombres y mujeres del tercer milenio. En efecto, las computadoras constituyen un formidable auxiliar de la memoria humana y pueden 
procesar datos con mucha mayor velocidad y precisión de la que solemos hacerlo los seres humanos, pueden calcular la probabilidad de ocurrencia de un fenómeno y hacer deducciones lógicas con una enorme cantidad de datos, pero lo que no pueden hacer es generar respuestas cuyos fundamentos no estén en su banco de memoria y para las que no han sido programadas. Tal es el rol del intelecto humano, por lo menos durante las primeras décadas del siglo XXI.

La necesidad de investigar acerca de la creatividad es, pues, ostensible y a ella han respondido autores como Craig y Manis (1962) y Flescher (1968), citados por Oerter (1975); Torrance (1963) y Getzels y Jakcson (1963), citados por Mayer (1986); Edwards Torrance (1972), citado por Espriu (1993); Wallach (1985), citado por Hoffman, París y Hall (1998); Moreno (1992), Guencer y Oral (1993), Olaseinde (1994), Kim y Michael (1995), Garaigordobil y Torres (1996), Khanam y Sen (1998), Lui-Lam (1998), Ortega (1999), Valenciano (1999) y Granados (2001). Pero hay aún muchos vacíos en el conocimiento y no se conocen estudios que hayan relacionado la variable nutrición con la variable creatividad.

En este contexto es que surgió la necesidad de responder al siguiente problema:

¿Qué efectos produce el déficit nutricional en la creatividad de los alumnos que cursan educación primaria en las escuelas de Lima metropolitana?

\section{ObJETIVO GENERAL}

- Conocer los efectos del déficit nutricional en la creatividad de los alumnos que cursan educación primaria en Lima metropolitana.

\section{OBJETIVOS ESPECÍFICOS}

- Describir y analizar las características de la creatividad de los niños con déficit nutricional con las de los niños adecuadamente nutridos.

- Comparar el rendimiento de los niños con déficit nutricional con el de los niños adecuadamente nutridos en una prueba de creatividad.

\section{HiPÓTESIS CENTRAL}

La creatividad de los niños que cursan educación primaria es afectada negativamente por el déficit nutricional.

\section{VARIABLES}

\section{Variable independiente}

La variable independiente es el estado nutricional. Se consideró esta como una variable dicotómica: déficit nutricional crónico y adecuada nutrición. Los indicadores de la variable estado nutricional fueron peso y talla. El diagnóstico nutricional fue efectuado por el equipo del área de nutrición del Centro de Salud del distrito de Pachacámac, dirigido por la magíster Olga Blas. 


\section{Variable dependiente}

La variable dependiente es la creatividad. Los indicadores de esta variable son: fluidez, flexibilidad, originalidad y organización.

\section{Variables de control}

Las variables de control consideradas fueron:

1. Grado de instrucción: tercero, cuarto, quinto y sexto grados de instrucción primaria

2. Sexo: varones y mujeres

3. Edad: de ocho a doce años.

\section{Método}

El estudio realizado se inscribe dentro de las investigaciones sustantivas. El método básico empleado en el estudio es el método descriptivo, debido a que la investigadora observó las variables, tal y como se presentaron en la realidad, comparando la creatividad de niños con déficit nutricional respecto a niños adecuadamente nutridos, precisando el grado de instrucción, edad y sexo. Se empleo un diseño causal comparativo.

\section{SuJETos}

La población objetivo estuvo constituida por 5.579 niños que cursan educación primaria en colegios estatales diurnos, en el sistema escolarizado del distrito de Pachacámac. La población accesible estuvo constituida por 3.987 alum- nos con las mismas características, que cursaban de tercero a sexto grado de educación primaria. Se eligió tal población debido a que, según el Primer Censo Nacional de Talla en Escolares un $60 \%$ de los 42 distritos de Lima metropolitana presentan una prevalencia de desnutrición crónica que va del $17 \%$ al un $39 \%$. El distrito que presenta el nivel más alto de desnutrición es Pachacámac (Unicef, Ministerio de Educación y otros, 1994). De la población accesible se tomó una muestra de 644 alumnos, que constituye el $16,15 \%$ de esta y el $11,54 \%$ de la población objetivo.

El muestreo se realizó en forma intencional y por cuotas (esto último para cada grado de instrucción), se constituyeron dos grupos apareados; es decir, para cada sujeto en A (con déficit nutricional) había un sujeto en B (adecuadamente nutrido); siendo los criterios de apareamiento: grado de instrucción, sexo y edad. En total fueron 322 sujetos con déficit nutricional y 322 sujetos adecuadamente nutridos.

\section{TÉCNICA E INSTRUMENTOS}

\section{Evaluación nutricional}

\section{Técnica básica: Observación indirecta} Instrumentos:

- Ficha de matrícula: para determinar la edad exacta y el sexo.

- Balanza de precisión: para determinar el peso exacto de cada sujeto. 
- Tallímetro: para determinar la estatura.

- Tablas elaboradas por el National Center for Health Statics (NCHS) recomendadas por la OMS y la OPS; estas tablas fueron utilizadas en la Encuesta Nacional de Nutrición y Salud (ENNSA) (Inide, 1986), en el Primer Censo Nacional de Talla en Escolares (Unicef, 1993) y en la Encuesta Demográfica y de Salud Familiar 1996 (Unicef, 1997).

- El software ANA, elaborado por la ONG Prisma, que permite un diagnóstico inmediato sobre la base de los indicadores nutricionales.

\section{Evaluación DE LA CREATIVIDAD}

La creatividad fue estimada con la prueba para evaluar indicadores básicos de creatividad (EIBC). Esta prueba fue propuesta por Sánchez (1991), quien trabajó con niños de 9 a 12 años de edad de Lima metropolitana. El citado autor realizó una adaptación de reactivos extraídos de otros instrumentos que evalúan la creatividad, como Wallach y Kohan (1965), Torrance (1965) y Guilford (1961). Esta prueba fue adaptada en 1995, con una muestra mucho más grande (Sánchez, 1995). El objetivo de esta prueba consiste en explorar y evaluar los indicadores básicos más importantes que caracterizan el comportamiento creativo de niños cuyas edades oscilan entre los 8 y los 12 años de edad. Entre estos indicadores destacan: la flui- dez, la originalidad, la flexibilidad y la organización.

La confiabilidad hallada por Sánchez (1995) se realizó sometiendo la prueba de creatividad al método de correlación por mitades, obteniéndose un coeficiente de fiabilidad de 0.84. Posteriormente, la prueba fue usada por Lui Lam (1998) y por Matalinares y Lazarte (2002), quienes trabajaron con una muestra de 735 niños, obteniendo un coeficiente de confiabilidad general de 0.9144 . Usando el estadístico Coeficiente Alfa de Cronbach, con un nivel de confianza $\mathrm{p}<0.05$, al analizar componente por componente se encontró que el coeficiente de confiabilidad para el componente fluidez fue de 0.8492 , el coeficiente de confiabilidad para el componente flexibilidad fue de 0.8652 , el coeficiente de confiabilidad para el componente originalidad fue de $0.8113 \mathrm{y}$, por último, el coeficiente de confiabilidad para el componente organización fue de 0.7374 .

El que los autores de la prueba hayan extraído los reactivos del instrumento que evalúan la creatividad, le confieren de facto validez de constructo al instrumento. Además, se trabajó la validez de la prueba a través del análisis factorial de sus diferentes componentes. Se encontró que existe un solo factor en las diversas áreas propuestas para la prueba, que permiten explicar el $88,23 \%$ de la varianza, este factor es la creatividad (Matalinares \& Lazarte, 2002). 
Tabla 1

Valores normativos de la escala de indicadores básicos de creatividad

\begin{tabular}{lccccc}
\hline Niveles & Fluidez & Flexibilidad & Originalidad & Organización & General \\
\hline Alto & $61 \mathrm{a}+$ & $23 \mathrm{a}+$ & $5 \mathrm{a}+$ & $12 \mathrm{a}+$ & $97 \mathrm{a}+$ \\
Medio & $25 \mathrm{a} 60$ & $9 \mathrm{a} \mathrm{22}$ & $2 \mathrm{a} 4$ & $6 \mathrm{a} 11$ & $44 \mathrm{a} \mathrm{96}$ \\
Bajo & $0 \mathrm{a} 24$ & $0 \mathrm{a} 8$ & $0 \mathrm{a} 1$ & $0 \mathrm{a} 5$ & $0 \mathrm{a} \mathrm{43}$ \\
\hline
\end{tabular}

En la tabla 1 se muestran los valores esperados en la prueba, en cada uno de sus indicadores y en general. Los datos se obtuvieron con una muestra de 735 sujetos de ambos sexos, de tercer a sexto grados de educación primaria, cuyas edades oscilaban entre ocho y doce años, y de estatus socioeconómicos medio y bajo procedentes de cole- gios estatales y particulares de Lima metropolitana. Los valores se obtuvieron con la media y la desviación estándar. El nivel medio se estableció con dos desviaciones estándar. Los niveles alto y bajo a partir de los límites superior e inferior del intervalo resultante respectivamente.

\section{Resultados}

Tabla 2

Frecuencias y porcentajes por niveles para el indicador fluidez en ambos grupos

\begin{tabular}{lcccccc}
\hline $\begin{array}{c}\text { Niveles } \\
\text { de } \\
\text { creatividad }\end{array}$ & \multicolumn{3}{c}{$\begin{array}{c}\text { Adeacuadamente } \\
\text { nutridos }\end{array}$} & & \multicolumn{3}{c}{$\begin{array}{c}\text { Déficit nutricional } \\
\text { crónico }\end{array}$} \\
\hline Bajo & $\mathrm{f}$ & $\mathrm{H}$ & $\mathrm{H}$ & $\mathrm{f}$ & $\mathrm{h}$ & $\mathrm{H}$ \\
Medio & 34 & 10,6 & 10,6 & 37 & 11,5 & 11,5 \\
Alto & 254 & 78,9 & 89,5 & 250 & 77,6 & 89,1 \\
\hline Total & 34 & 10,6 & 100,0 & 35 & 10,9 & 100,0 \\
\hline
\end{tabular}

$f$ frecuencia de alumnos

h porcentaje de alumnos 
Tabla 3

Frecuencias y porcentajes por niveles en el indicador flexibilidad en ambos grupos

\begin{tabular}{lcccccc}
\hline $\begin{array}{c}\text { Niveles } \\
\text { de } \\
\text { creatividad }\end{array}$ & \multicolumn{3}{c}{$\begin{array}{c}\text { Adeacuadamente } \\
\text { nutridos }\end{array}$} & H & \multicolumn{3}{c}{$\begin{array}{c}\text { Déficit nutricional } \\
\text { crónico }\end{array}$} \\
\hline Bajo & $\mathrm{f}$ & $\mathrm{H}$ & $\mathrm{H}$ & $\mathrm{h}$ & $\mathrm{H}$ \\
Medio & 286 & 88,8 & 88,8 & 298 & 92,5 & 92,5 \\
Alto & 35 & 10,9 & 99,7 & 24 & 7,5 & 100,0 \\
\hline Total & 1 & 0,3 & 100,0 & 0 & 0 & 100,0 \\
\hline
\end{tabular}

$f$ frecuencia de alumnos

h porcentaje de alumnos

Tabla 4

Frecuencias y porcentajes por niveles en el indicador originalidad en ambos grupos

\begin{tabular}{lcccccc}
\hline $\begin{array}{c}\text { Niveles } \\
\text { de } \\
\text { creatividad }\end{array}$ & \multicolumn{3}{c}{$\begin{array}{c}\text { Adeacuadamente } \\
\text { nutridos }\end{array}$} & & \multicolumn{3}{c}{$\begin{array}{c}\text { Déficit nutricional } \\
\text { crónico }\end{array}$} \\
\hline Bajo & $\mathrm{F}$ & $\mathrm{H}$ & $\mathrm{H}$ & $\mathrm{f}$ & $\mathrm{h}$ & $\mathrm{H}$ \\
Medio & 197 & 61,2 & 61,2 & 221 & 68,6 & 68,6 \\
Alto & 92 & 28,6 & 89,8 & 79 & 24,5 & 93,2 \\
\hline Total & 33 & 10,2 & 100,0 & 22 & 6,8 & 100,0 \\
\hline
\end{tabular}

$f$ frecuencia de alumnos

h porcentaje de alumnos

Tabla 5

Frecuencias y porcentajes por niveles en el indicador organización de ambos grupos

\begin{tabular}{lcccccc}
\hline $\begin{array}{c}\text { Niveles } \\
\text { de } \\
\text { creatividad }\end{array}$ & \multicolumn{3}{c}{$\begin{array}{c}\text { Adeacuadamente } \\
\text { nutridos }\end{array}$} & H & \multicolumn{3}{c}{$\begin{array}{c}\text { Déficit nutricional } \\
\text { crónico }\end{array}$} \\
\hline Bajo & 52 & 16,1 & 16,1 & $\mathrm{f}$ & $\mathrm{h}$ & $\mathrm{H}$ \\
Medio & 243 & 75,5 & 91,6 & 48 & 14,9 & 14,9 \\
Alto & 27 & 8,4 & & 265 & 82,3 & 97,2 \\
\hline Total & 322 & 100,00 & & 9 & 2,8 & 100,0 \\
\hline
\end{tabular}

$f$ frecuencia de alumnos

h porcentaje de alumnos 
Tabla 6

Frecuencias y porcentajes por niveles en creatividad general de ambos grupos

\begin{tabular}{lcccccc}
\hline $\begin{array}{c}\text { Niveles } \\
\text { de } \\
\text { creatividad }\end{array}$ & \multicolumn{3}{c}{$\begin{array}{c}\text { Adeacuadamente } \\
\text { nutridos }\end{array}$} & H & \multicolumn{3}{c}{$\begin{array}{c}\text { Déficit nutricional } \\
\text { crónico }\end{array}$} \\
\hline Bajo & $\mathrm{f}$ & $\mathrm{H}$ & $\mathrm{H}$ & $\mathrm{f}$ & $\mathrm{h}$ & $\mathrm{H}$ \\
Medio & 100 & 31,1 & 31,1 & 111 & 34,5 & 34,5 \\
Alto & 216 & 67,1 & 98,2 & 206 & 64,0 & 98,5 \\
\hline Total & 6 & 1,9 & 100,0 & 5 & 1,6 & 100,0 \\
\hline
\end{tabular}

$f$ frecuencia de alumnos

h porcentaje de alumnos

Tabla 7

Medias de puntajes en los indicadores de creatividad de los alumnos según el grado de instrucción

\begin{tabular}{lcccccccccc}
\hline Grado de & \multicolumn{2}{c}{ Fluidez } & \multicolumn{2}{c}{ Flexibilidad } & \multicolumn{2}{c}{ Originalidad } & \multicolumn{2}{c}{ Organización } & \multicolumn{2}{c}{ General } \\
instruc. & $\overline{\mathrm{X}}_{1}$ & $\overline{\mathrm{X}}_{2}$ & $\overline{\mathrm{X}}_{1}$ & $\overline{\mathrm{X}}_{2}$ & $\overline{\mathrm{X}}_{1}$ & $\overline{\mathrm{X}}_{2}$ & $\overline{\mathrm{X}}_{1}$ & $\overline{\mathrm{X}}_{2}$ & $\overline{\mathrm{X}}_{1}$ & $\overline{\mathrm{X}}_{2}$ \\
\hline Tercero & 37,34 & 36,78 & 3,07 & 4,03 & 6,72 & 6,38 & 1,00 & 0,79 & 48,13 & 47,98 \\
Cuarto & 36,54 & 38,41 & 3,10 & 2,77 & 8,06 & 8,21 & 1,08 & 1,76 & 50,65 & 51,15 \\
Quinto & 39,10 & 43,47 & 2,79 & 3,20 & 8,14 & 8,75 & 2,03 & 2,45 & 52,06 & 57,87 \\
Sexto & 50,74 & 46,39 & 3,57 & 3,26 & 8,07 & 9,13 & 1,67 & 1,88 & 64,05 & 60,66 \\
\hline
\end{tabular}

$\mathrm{N} 644$

$\overline{\mathrm{X}}_{1}$ Media de alumnos con déficit nutricional crónico

$\overline{\mathrm{X}}_{2}$ Media de alumnos adecuadamente nutridos

Tabla 8

Medias de puntajes en los indicadores de creatividad de los alumnos según la edad

\begin{tabular}{lcccccccccc}
\hline Edad & \multicolumn{2}{c}{ Fluidez } & \multicolumn{2}{c}{ Flexibilidad } & \multicolumn{2}{c}{ Originalidad } & \multicolumn{2}{c}{ Organización } & \multicolumn{2}{c}{ General } \\
& $\bar{X}_{1}$ & $\bar{x}_{2}$ & $\bar{X}_{1}$ & $\bar{X}_{2}$ & $\bar{X}_{1}$ & $\bar{x}_{2}$ & $\bar{x}_{1}$ & $\bar{x}_{2}$ & $\bar{X}_{1}$ & $\bar{X}_{2}$ \\
\hline Ocho & 37,66 & 36,63 & 3,29 & 4,12 & 1,00 & 0,92 & 7,05 & 7,05 & 49,00 & 48,72 \\
Nueve & 37,00 & 39,70 & 3,31 & 3,08 & 1,42 & 1,73 & 7,85 & 7,88 & 49,58 & 52,39 \\
Diez & 41,02 & 41,91 & 3,12 & 2,81 & 1,58 & 1,93 & 7,98 & 8,72 & 53,7 & 55,37 \\
Once & 46,17 & 45,10 & 3,17 & 3,16 & 1,80 & 2,44 & 8,31 & 9,00 & 59,45 & 59,7 \\
Doce & 42,88 & 43,39 & 2,39 & 3,14 & 1,57 & 1,52 & 7,68 & 8,32 & 54,62 & 56,37 \\
\hline
\end{tabular}

N 644

$\bar{X}_{1}$ Media de alumnos con déficit nutricional crónico

$\overline{\mathrm{X}}_{2}$ Media de alumnos adecuadamente nutridos 
Tabla 9

Niveles de creatividad en la muestra de niños con déficit nutricional por grado de instrucción

\begin{tabular}{|c|c|c|c|c|c|c|c|c|c|c|}
\hline Grados & & & Tercero & & & & & Cuarto & & \\
\hline $\begin{array}{l}\text { Niveles } \\
\text { Alto }\end{array}$ & Fluid. & Flex. & Orig. & Org. & Gral. & Fluid. & Flex. & Orig. & Org. & Gral. \\
\hline Medio & $x$ & & & & $x$ & $x$ & & & & $x$ \\
\hline Bajo & & $x$ & $x$ & $x$ & & & $x$ & $x$ & $x$ & \\
\hline Grados & & & Quinto & & & & & Sexto & & \\
\hline $\begin{array}{l}\text { Niveles } \\
\text { Alto }\end{array}$ & Fluid. & Flex. & Orig. & Org. & Gral. & Fluid. & Flex. & Orig. & Org. & Gral. \\
\hline Medio & $X$ & & & & $x$ & $x$ & & & & $x$ \\
\hline Bajo & & $x$ & $x$ & $x$ & & & $x$ & $x$ & $x$ & \\
\hline
\end{tabular}

Tabla 10

Niveles de creatividad en la muestra de niños adecuadamente nutridos por grado de instrucción

\begin{tabular}{|c|c|c|c|c|c|c|c|c|c|c|}
\hline Grados & & & Tercero & & & & & Cuarto & & \\
\hline $\begin{array}{l}\text { Niveles } \\
\text { Alto }\end{array}$ & Fluid. & Flex. & Orig. & Org. & Gral. & Fluid. & Flex. & Orig. & Org. & Gral. \\
\hline Medio & $X$ & & & & $X$ & $x$ & & & & $X$ \\
\hline Bajo & & $x$ & $X$ & $X$ & & & $x$ & $x$ & $x$ & \\
\hline Grados & & & Quinto & & & & & Sexto & & \\
\hline $\begin{array}{l}\text { Niveles } \\
\text { Alto }\end{array}$ & Fluid. & Flex. & Orig. & Org. & Gral. & Fluid. & Flex. & Orig. & Org. & Gral. \\
\hline Medio & $x$ & & & & $x$ & $x$ & & & & $x$ \\
\hline Bajo & & $x$ & $x$ & $x$ & & & $x$ & $x$ & $x$ & \\
\hline
\end{tabular}

Tabla 11

Niveles de creatividad en la muestra de niños con déficit nutricional por sexo

\begin{tabular}{lcccccccccc}
\hline Grados & \multicolumn{4}{c}{ Femenino } \\
\hline $\begin{array}{l}\text { Niveles } \\
\text { Alto }\end{array}$ & Fluid. & Flex. & Orig. & Org. & Gral. & Fluid. & Flex. & Orig. & Org. & Gral. \\
Medio & $\mathrm{X}$ & & $\mathrm{X}$ & $\mathrm{X}$ & $\mathrm{X}$ & $\mathrm{X}$ & & $\mathrm{X}$ & $\mathrm{X}$ & $\mathrm{X}$ \\
Bajo & & $\mathrm{X}$ & & & & & $\mathrm{X}$ & & & \\
\hline
\end{tabular}


Tabla 12

Cálculo de la prueba de Kolmokorov-Smirnov para la variable creatividad general

\begin{tabular}{ccc}
\hline & Índice Kolmogorov-Smirnov & $\mathrm{P}$ \\
\hline $\begin{array}{l}\text { Creatividad } \\
\text { general }\end{array}$ & .433 & .992 \\
\hline $\mathrm{p}<.05$ & \\
$\mathrm{~N} 644$ &
\end{tabular}

Tabla 13

Resultados en creatividad total de los alumnos adecuadamente nutridos y con déficit nutricional crónico

\begin{tabular}{|c|c|c|c|c|c|c|}
\hline & $\begin{array}{c}\text { Adecuadamente } \\
\text { nutridos }\end{array}$ & \multicolumn{2}{|c|}{$\begin{array}{l}\text { Déficit nutricional } \\
\text { crónico }\end{array}$} & \multirow[t]{2}{*}{$\mathrm{zt}$} & \multirow[t]{2}{*}{$z p$} & \multirow[t]{2}{*}{ Diagnóstico } \\
\hline $\bar{x}_{1}$ & $\mathrm{~S}_{1}$ & $\bar{x}_{2}$ & $S_{2}$ & & & \\
\hline Creatividad total 52.83 & 18.50 & 54.07 & 18.11 & 1,96 & 0.865 & $\begin{array}{c}\text { No } \\
\text { significativo }\end{array}$ \\
\hline $\begin{array}{ll}p<.05 \\
N & 644 \\
\bar{X}_{1} & \text { Media de alumnos } \\
\bar{X}_{2} & \text { Media de alumnos } \\
\mathrm{S}_{1} & \text { Desviación estánda } \\
\mathrm{S}_{2} & \text { Desviación estánda }\end{array}$ & $\begin{array}{l}\text { n déficit } \\
\text { decuada } \\
\text { de alum } \\
\text { de alum }\end{array}$ & $\begin{array}{l}\text { ional cró } \\
\text { nutridos } \\
\text { n déficit } \\
\text { lecuadar }\end{array}$ & $\begin{array}{l}\text { co } \\
\text { utriciona } \\
\text { nte nut }\end{array}$ & & & \\
\hline
\end{tabular}

Tabla 14

Resultados en los Indicadores de Creatividad de los alumnos adecuadamente nutridos y con déficit nutricional crónico

\begin{tabular}{lrrrrrrr}
\hline $\begin{array}{l}\text { Indicadores } \\
\text { de creatividad }\end{array}$ & $\begin{array}{c}\text { Adecuadamente } \\
\text { nutridos }\end{array}$ & \multicolumn{2}{c}{$\begin{array}{c}\text { Déficit nutricional } \\
\text { crónico }\end{array}$} & zt & zp & Diagnóstico \\
\hline & $\overline{\mathrm{X}}_{1}$ & \multicolumn{1}{c}{$\mathrm{S}_{1}$} & \multicolumn{1}{c}{$\overline{\mathrm{X}}_{2}$} & \multicolumn{1}{c}{$\mathrm{S}_{2}$} & & & \\
\hline Fluidez & 40.98 & 14.35 & 40.52 & 15.03 & 1,96 & .400 & No significativo \\
Flexibilidad & 3.29 & 3.73 & 3.12 & 3.62 & 1,96 & .558 & No significativo \\
Originalidad & 1.71 & 2.14 & 1.42 & 2.07 & 1,96 & 1.779 & No significativo \\
Organización & 8.10 & 2.62 & 7.77 & 2.35 & 1,96 & 1.676 & No significativo \\
\hline $\mathrm{p}<.05$ & & & & & & & \\
$\mathrm{~N} 644$ & & & & & & &
\end{tabular}


Tabla 15

Resultados en el indicador fluidez de los alumnos adecuadamente nutridos y con déficit nutricional crónico en función del grado de instrucción

\begin{tabular}{lccccrrr}
\hline $\begin{array}{l}\text { Grado de } \\
\text { intrucción }\end{array}$ & $\begin{array}{c}\text { Adecuadamente } \\
\text { nutridos }\end{array}$ & \multicolumn{2}{c}{$\begin{array}{c}\text { Déficit nutricional } \\
\text { crónico }\end{array}$} & zt & zp & Diagnóstico \\
\hline & $\overline{\mathrm{X}}_{1}$ & $\mathrm{~S}_{1}$ & $\overline{\mathrm{X}}_{2}$ & $\mathrm{~S}_{2}$ & & & \\
\hline Tercero & 36.78 & 11.83 & 37.34 & 12.73 & 1,96 & -.283 & No significativo \\
Cuarto & 38.41 & 13.89 & 36.54 & 13.51 & 1,96 & .954 & No significativo \\
Quinto & 43.47 & 16.40 & 39.10 & 13.25 & 1,96 & 1.806 & No significativo \\
Sexto & 46.39 & 13.14 & 50.74 & 16.57 & 1,96 & -1.744 & No significativo \\
\hline
\end{tabular}

Tabla 16

Resultados en el indicador flexibilidad de los alumnos adecuadamente nutridos y con déficit nutricional crónico en función del grado de instrucción

\begin{tabular}{lccccccc}
\hline $\begin{array}{l}\text { Grado de } \\
\text { intrucción }\end{array}$ & $\begin{array}{c}\text { Adecuadamente } \\
\text { nutridos }\end{array}$ & \multicolumn{2}{c}{$\begin{array}{c}\text { Déficit nutricional } \\
\text { crónico }\end{array}$} & zt & zp & Diagnóstico \\
\hline & $\overline{\mathrm{X}}_{1}$ & $\mathrm{~S}_{1}$ & $\overline{\mathrm{X}}_{2}$ & $\mathrm{~S}_{2}$ & & & \\
\hline Tercero & 4.03 & 4.40 & 3.07 & 3.72 & 1,96 & 1.459 & No significativo \\
Cuarto & 2.77 & 2.95 & 3.10 & 2.88 & 1,96 & -.788 & No significativo \\
Quinto & 3.20 & 3.53 & 2.79 & 4.09 & 1,96 & .657 & No significativo \\
Sexto & 3.26 & 4.02 & 3.57 & 3.88 & 1,96 & -.464 & No significativo \\
\hline
\end{tabular}

Tabla 17

Resultados en el indicador originalidad de los alumnos adecuadamente nutridos y con déficit nutricional crónico en función del grado de instrucción

\begin{tabular}{lcccrrrr}
\hline $\begin{array}{l}\text { Grado de } \\
\text { intrucción }\end{array}$ & $\begin{array}{c}\text { Adecuadamente } \\
\text { nutridos }\end{array}$ & \multicolumn{2}{c}{$\begin{array}{c}\text { Déficit nutricional } \\
\text { crónico }\end{array}$} & zt & zp & Diagnóstico \\
\hline & $\overline{\mathrm{X}}_{1}$ & $\mathrm{~S}_{1}$ & $\overline{\mathrm{X}}_{2}$ & $\mathrm{~S}_{2}$ & & & \\
\hline Tercero & .79 & 1.14 & 1.00 & 1.14 & 1,96 & -1.113 & No significativo \\
Cuarto & 1.76 & 2.12 & 1.08 & 1.30 & 1,96 & 2.697 & Significativo \\
Quinto & 2.45 & 2.68 & 2.03 & 3.29 & 1,96 & .876 & No significativo \\
Sexto & 1.88 & 2.04 & 1.67 & 1.82 & 1,96 & .647 & No significativo \\
\hline
\end{tabular}


Tabla 18

Resultados en el indicador organización de los alumnos adecuadamente nutridos y con déficit nutricional crónico en función del grado de instrucción

\begin{tabular}{lccccccc}
\hline $\begin{array}{l}\text { Grado de } \\
\text { intrucción }\end{array}$ & $\begin{array}{c}\text { Adecuadamente } \\
\text { nutridos }\end{array}$ & $\begin{array}{c}\text { Déficit nutricional } \\
\text { crónico }\end{array}$ & zt & zp & Diagnóstico \\
\hline & $\overline{\mathrm{X}}_{1}$ & $\mathrm{~S}_{1}$ & $\overline{\mathrm{X}}_{2}$ & $\mathrm{~S}_{2}$ & & & \\
\hline Tercero & 6.38 & 2.67 & 6.72 & 2.70 & 1,96 & -.784 & No significativo \\
Cuarto & 8.21 & 2.43 & 8.06 & 2.18 & 1,96 & .435 & No significativo \\
Quinto & 8.75 & 2.47 & 8.14 & 2.32 & 1,96 & 1.553 & No significativo \\
Sexto & 9.13 & 2.06 & 8.07 & 1.90 & 1,96 & 3.193 & Significativo \\
\hline
\end{tabular}

Tabla 19

Resultados en el indicador fluidez de los alumnos adecuadamente nutridos y con déficit nutricional crónico en función de su edad

\begin{tabular}{lcccrrrr}
\hline Edad & $\begin{array}{c}\text { Adecuadamente } \\
\text { nutridos }\end{array}$ & \multicolumn{2}{c}{$\begin{array}{c}\text { Déficit nutricional } \\
\text { crónico }\end{array}$} & zt & zp & Diagnóstico \\
\hline & $\overline{\mathrm{X}}_{1}$ & $\mathrm{~S}_{1}$ & $\overline{\mathrm{X}}_{2}$ & \multicolumn{1}{c}{$\mathrm{s}_{2}$} & & & \\
\hline Ocho & 36.63 & 12.35 & 37.66 & 13.03 & 1,96 & -.474 & No significativo \\
Nueve & 39.70 & 13.96 & 37.00 & 13.64 & 1,96 & 1.284 & No significativo \\
Diez & 41.91 & 14.30 & 41.02 & 14.29 & 1,96 & .361 & No significativo \\
Once & 45.10 & 13.96 & 46.17 & 16.51 & 1,96 & -.392 & No significativo \\
Doce & 43.39 & 17.17 & 42.88 & 17.06 & 1,96 & .134 & No significativo \\
\hline
\end{tabular}

Tabla 20

Resultados en el indicador flexibilidad de los alumnos adecuadamente nutridos y con déficit nutricional crónico en función de su edad

\begin{tabular}{lccccccc}
\hline Edad & $\begin{array}{c}\text { Adecuadamente } \\
\text { nutridos }\end{array}$ & \multicolumn{2}{c}{$\begin{array}{c}\text { Déficit nutricional } \\
\text { crónico }\end{array}$} & zt & zp & Diagnóstico \\
\hline & $\overline{\mathrm{X}}_{1}$ & $\mathrm{~S}_{1}$ & $\overline{\mathrm{X}}_{2}$ & $\mathrm{~S}_{2}$ & & & \\
\hline Ocho & 4.12 & 4.46 & 3.29 & 3.60 & 1,96 & 1.184 & No significativo \\
Nueve & 3.08 & 3.22 & 3.31 & 3.60 & 1,96 & -.445 & No significativo \\
Diez & 2.81 & 3.96 & 3.12 & 3.91 & 1,96 & -.462 & No significativo \\
Once & 3.16 & 3.15 & 3.17 & 3.92 & 1,96 & -0.12 & No significativo \\
Doce & 3.14 & 3.75 & 2.39 & 2.31 & 1,96 & .792 & No significativo \\
\hline
\end{tabular}


Tabla 21

Resultados en el indicador originalidad de los alumnos adecuadamente nutridos y con déficit nutricional crónico en función de su edad

\begin{tabular}{lccccccc}
\hline Edad & $\begin{array}{c}\text { Adecuadamente } \\
\text { nutridos }\end{array}$ & \multicolumn{2}{c}{$\begin{array}{c}\text { Déficit nutricional } \\
\text { crónico }\end{array}$} & zt & zp & Diagnóstico \\
\hline & $\overline{\mathrm{X}}_{1}$ & $\mathrm{~S}_{1}$ & $\overline{\mathrm{X}}_{2}$ & $\mathrm{~S}_{2}$ & & & \\
\hline Ocho & .92 & 1.22 & 1.00 & 1.29 & 1,96 & -.787 & No significativo \\
Nueve & 1.73 & 2.25 & 1.42 & 2.51 & 1,96 & .868 & No significativo \\
Diez & 1.93 & 2.46 & 1.58 & 2.49 & 1,96 & .815 & No significativo \\
Once & 2.44 & 2.33 & 1.80 & 1.90 & 1,96 & 1.704 & No significativo \\
Doce & 1.52 & 1.72 & 1.57 & 1.93 & 1,96 & -0.75 & No significativo \\
\hline
\end{tabular}

Tabla 22

Resultados en el indicador organización de los alumnos adecuadamente nutridos y con déficit nutricional crónico en función de su edad

\begin{tabular}{lccccccc}
\hline Edad & $\begin{array}{c}\text { Adecuadamente } \\
\text { nutridos }\end{array}$ & \multicolumn{2}{c}{$\begin{array}{c}\text { Déficit nutricional } \\
\text { crónico }\end{array}$} & zt & zp & Diagnóstico \\
\hline & $\overline{\mathrm{X}}_{1}$ & $\mathrm{~s}_{1}$ & $\overline{\mathrm{X}}_{2}$ & \multicolumn{1}{c}{$\mathrm{s}_{2}$} & & & \\
\hline Ocho & 7.05 & 2.75 & 7.05 & 2.43 & 1,96 & .002 & No significativo \\
Nueve & 7.88 & 2.46 & 7.85 & 2.43 & 1,96 & .084 & No significativo \\
Diez & 8.72 & 2.27 & 7.98 & 2.43 & 1,96 & 1.794 & No significativo \\
Once & 9.00 & 2.37 & 8.31 & 1.82 & 1,96 & 1.827 & No significativo \\
Doce & 8.32 & 2.91 & 7.68 & 2.34 & 1,96 & 1.074 & No significativo \\
\hline
\end{tabular}

Tabla 23

Resultados de la comparación de los indicadores de creatividad según el sexo en población de alumnos con déficit nutricional crónico

\begin{tabular}{lrrrrrrr}
\hline $\begin{array}{l}\text { Indicadores } \\
\text { de creatividad }\end{array}$ & \multicolumn{2}{c}{ Varones } & \multicolumn{2}{c}{ Mujeres } & zt & zp & Diagnóstico \\
\hline & $\overline{\mathrm{X}}_{1}$ & \multicolumn{1}{c}{$\mathrm{S}_{1}$} & \multicolumn{1}{c}{$\overline{\mathrm{X}}_{2}$} & \multicolumn{1}{c}{$\mathrm{S}_{2}$} \\
\hline Fluidez & 42.07 & 16.00 & 38.84 & 13.76 & 1,96 & 1.968 & Significativo \\
Flexibilidad & 3.05 & 3.56 & 3.21 & 3.69 & 1,96 & -.392 & No significativo \\
Originalidad & 1.39 & 2.32 & 1.45 & 1.77 & 1,96 & -.272 & No significativo \\
\hline Organización & 7.50 & 2.35 & 8.05 & 2.33 & 1,96 & -2.01 & Significativo \\
\hline
\end{tabular}




\section{DisCUSIÓN}

Los resultados expuestos han conducido, en términos generales, a establecer que en casi todas las comparaciones no existen diferencias entre los niños con déficit nutricional crónico y el grupo de contraste, excepto en dos grupos: en cuarto y sexto grados, en los indicadores originalidad y organización, respectivamente; en ambos casos los adecuadamente nutridos obtuvieron medias significativamente superiores a las de los niños con déficit nutricional crónico (tablas 17 y 18). Por otro lado, al comparar varones contra mujeres con déficit nutricional, se encontró que los varones obtuvieron una media estadísticamente superior en fluidez, mientras que las mujeres lograron una media superior en organización (tabla 23).

De otra parte, se han podido establecer las variaciones de las medias y sus correspondientes niveles en función del grado de instrucción y la edad en cada uno de los indicadores de creatividad (tablas 7, 8, 9 y 10), descubriéndose tendencias ascendentes en ciertos casos, oscilaciones en otros, y, lo que es más notable aún, caídas en el último grado y la más alta edad, en varios casos. Datos que refuerzan los hallazgos de Torrance (1983) y Dacey (1989) citados por Espriu (1993), quienes también hallaron caídas en los indicadores de creatividad en ciertos momentos del desarrollo, y aunque tales caídas -en las investigaciones de los referidos autores- han sido reportadas en momentos diferentes de los que aquí se han observado, podrían contribuir a sustentar la idea de que hay períodos en el desarrollo humano en que -ya sea por la educación, ya por otros factoreslos índices de creatividad disminuirían. De hecho, Torrance ha propuesto la idea de que se trata de etapas en las que operan en forma contundente presiones externas autoritarias, decrementando la motivación en los niños.

Pero, ¿qué significan todos estos resultados en términos cualitativos? ¿De qué manera pueden ser interpretados estos hallazgos?

Naturalmente, lo que salta a la vista es que el estado nutricional, en sí mismo, no parece ser una variable demasiado importante en la diferenciación de la creatividad de los estudiantes de educación primaria. Dicho en otros términos, la creatividad podría no ser afectada por el déficit nutricional de los niños, lo cual significaría que estos pueden ser más o menos creativos, independientemente de su estado nutricional. En efecto, el hecho de que en todas las comparaciones realizadas entre los grupos y subgrupos - con y sin déficit nutricional (tablas 13 al 23)- solo se hayan registrado diferencias significativas en dos grupos (cuarto y sexto grados) (tablas 17 y 18), revelaría el escaso poder distintivo de la variable estado nutricional, por lo menos con respecto a la creatividad. Estos hallazgos refuerzan la idea de Torrance (1963), citado por Espriu (1993), según 
el cual la mayor responsabilidad en el desarrollo de la creatividad la tendrían los factores ambientales. En la misma dirección, Kattami, Elkaissi y Kattami (1995) han puesto énfasis en la importancia que tienen los factores sociales, económicos y educacionales sobre la creatividad. Además, no debe perderse de vista que Pollit (2002) ha señalado que en el desarrollo intelectual, más importante que el efecto del déficit nutricional, es el carácter de la estimulación sociocultural. Algo análogo se había encontrado ya al estudiar los efectos del déficit nutricional sobre la atención (Matalinares, 1989) y sobre la memoria (Yarlequé, 1989) así como de la psicomotricidad (Zavala, 1989; Ocrospoma y otros, 1991). Y, más recientemente, Deusa (1999) ha encontrado que el nivel de cultura y la condición socioeconómica de los padres se corresponde con los resultados de sus hijos en las pruebas de creatividad.

Ahora bien, el hecho de haber apareado los grupos brinda cierta seguridad de que son similares en muchas variables, excepto en el estado nutricional. Por consiguiente resulta legítimo suponer que las variables (socioculturales) en las que son semejantes, los grupos tienen más peso en los resultados que aquello en lo que se diferencian (estado nutricional). También esto fue reportado por Stoch y Smithe (1976), citado por Pollit (1984); Grantham \& Mc Gregor (1982), citado por Pollit (2002), y Klein (1983).
Sin embargo, es necesario detenerse un momento en las diferencias apuntadas. Desde luego, es difícil explicar un fenómeno aislado como el señalado. No obstante, la tabla 18 permite observar medias mayores en organización obtenidas por el grupo de adecuadamente nutridos, en todos los casos, aunque tales diferencias no alcanzan el nivel de significación estadística, excepto en el sexto grado; pese a que en sexto grado se registra descenso en ambos grupos. Es pertinente recordar que tales oscilaciones, así como el hecho de que no en todos los grados se registran diferencias entre adecuadamente nutridos y niños con déficit nutricional, fueron reportadas por Yarlequé (1989) y por Ocrospoma y otros (1991). Aunque no debe perderse de vista que estos hallazgos contradicen la tesis de Méndez y Adair (1991), citado por Pollit (2002), según la cual los efectos del déficit nutricional sobre la actividad intelectual disminuirían conforme avanza la edad. Pero ratifican la idea, expuesta líneas arriba, de que factores externos (como el grado de instrucción) son más importantes en la diferenciación de los individuos que el estado nutricional. Es posible también que algunas variables no controladas, presentes en la estimulación que reciben los estudiantes de la población en estudio, se hallen en la base de tales diferencias y que la organización sea más permeable a ella.

En este sentido, hay que admitir que el presente trabajo no brinda los ele- 
mentos necesarios para esclarecer a plenitud el fenómeno registrado y será necesario realizar nuevas investigaciones que abunden sobre el punto. Lo que sí es evidente es que todos los indicadores de la creatividad no varían de modo uniforme (tablas 7 y 8), con el grado de instrucción ni con la edad.

Ahora bien, no puede soslayarse que Moreno (1992), al estudiar la relación entre creatividad e inteligencia en una muestra de 615 niños de ambos sexos, ha planteado la idea de que el factor de elaboración, que aquí se denomina organización y que fue definido por Torrance como de creatividad, no formaría parte de esta capacidad del intelecto y que, en cualquier caso, sería más de inteligencia que de creatividad por sus repercusiones educativas. Es, pues, necesario seguir estudiando qué componentes son los que forman esta capacidad denominada creatividad. Pues de ser cierta la suposición de Moreno, nuestros hallazgos se encontrarían en la dirección de su trabajo. Más aun cuanto que investigadores como Getzels y Jakcson (1963), citados por Mayer (1986), y Flescher (1968), citado por Oerter (1975), han relacionado las realizaciones de inteligencia y de creatividad, encontrando que no hay correlación entre estas variables. Del mismo modo, Anastasi y Schaefer (1971), citados por Mayer (1986), trabajando con una muestra de 989 estudiantes universitarios de Nueva York, descubrieron bajas co- rrelaciones entre tests de creatividad y test de CI. Otro tanto fue reportado por Garaigordobil y Torres (1997). El propio Torrance (1972), citado por Espriu (1993), ha reportado resultados semejantes. Pese a los hallazgos de Wallach (1985) citado por Hoffman, París y Hall (1998), quien sí halló correlación entre ellas.

Hay que admitir que el punto es discutible. No obstante, si se admite la tesis de Moreno (1992) y el hecho de que la creatividad no se relaciona con la inteligencia, por lo menos en la mayoría de los casos, estos resultados serían en este punto explicables.

Otra es la situación cuando se observa la comparación entre varones y mujeres con déficit nutricional (tabla 23). Aquí los varones obtienen puntajes estadísticamente superiores en fluidez, pero las mujeres en organización. Si se toma en cuenta que ambos subgrupos estuvieron constituidos por niños con déficit nutricional, entonces parece evidente que los resultados están más asociados a las variables relacionadas con el género, que con diferencias biológicas. En efecto, si tales diferencias reflejasen las ventajas de un sexo con respecto al otro, entonces estas deberían hallarse en la misma dirección en ambos casos. Pero al no ser así, es lícito suponer que más que al sexo, las diferencias tengan en su base elementos propios del género. Los cuales evidentemente pueden tener distintos efectos en los subgrupos. De hecho, un 
trabajo realizado en Guatemala (Pollit, 2002) ha permitido plantear una serie de dudas respecto del papel que tiene el género en relación con el rendimiento intelectual. Estos hallazgos revelarían que el género y la creatividad se relacionan de modo distinto que el género y la memoria, ya que en los estudios de Yarlequé (1989) esta variable mostró ser írrita con respecto a la memoria.

De esto se desprendería que los fenómenos psíquicos superiores son afectados de modo diferencial por el género. Este asunto ha sido también abordado por García Jiménez y Yela (1994), pero la controversia no ha cesado. Los hallazgos de Kim y Michael (1995) y de Burgos (1999) han mostrado diferencias en creatividad entre varones y mujeres, con ventaja de estas sobre aquellos. Resultados diferentes fueron hallados por Khanam y Sen (1998). En este sentido, los resultados aquí expuestos contienen un aspecto novedoso, ya que pondrían de manifiesto que hay por lo menos un indicador de creatividad en el que los varones pueden ser superiores a las mujeres; esto es en fluidez. Empero, si retomamos la idea de Moreno (1992) -según la cual la organización no sería parte de la creatividad sino más bien de la inteligencia- tendríamos que admitir también que hay por lo menos un indicador de inteligencia en el que las mujeres, en ciertas condiciones, muestran ser superiores a los varones. Sin embargo, si mantenemos la idea de que la orga- nización es parte de la creatividad, entonces deberemos aceptar que las mujeres pueden ser más creativas que los varones, por lo menos en uno de los cuatro indicadores estudiados (organización).

Si se regresa por un momento a las oscilaciones a que se aludió al comenzar la discusión en los indicadores de la creatividad (tablas 7 y 8). Las tablas 9 y 10 muestran la similitud de performance entre ambos grupos, y -lo que es más notable aún- revelan niveles de creatividad general y en fluidez dentro de la norma. Pero ambos grupos se ubican por debajo de lo esperado en flexibilidad, organización y originalidad (tablas 9 y 10).

Además, se ha encontrado que en fluidez, organización y creatividad general la mayor frecuencia en ambos grupos tiende a acumularse en el nivel medio (tablas 2, 5 y 6), pero en flexibilidad y originalidad las frecuencias más altas se encuentran en el nivel bajo (tablas 3 y 4 ).

Esto significaría que los niños con y sin déficit nutricional tenderían a la rigidez y presentarían dificultades cuando las tareas exigen capacidad para reordenar lo realizado, para reestructurar y para organizar nuevas estructuras. Tendrían dificultad también para emitir ideas nuevas, poco frecuentes e ingeniosas (Sánchez, 2003). Es poco probable que tales dificultades sean un subproducto del déficit nutricional, pues de ser así estarían presentes en un grupo y 
no en el otro. Por consiguiente, resulta lícito achacar tales características a los factores propios de la educación formal e informal a que están expuestos los niños; por lo menos los de estatus socioeconómico bajo.

Así pues, estos hallazgos tienen implicancias psicológicas y pedagógicas, además de socioculturales. ¿Se está produciendo una generación de personas poco flexibles, tendientes al dogmatismo y con escasa capacidad de reorganizar y elaborar nuevas estructuras?

Los hallazgos aquí expuestos deben alertar a los responsables de la educación en el Perú, ya que en un mundo de constantes cambios y renovación, una generación de gente poco flexible, original y con escasa capacidad de organización tendría también muy pocas posibilidades de éxito.

En lo concerniente a la originalidad hay que recordar que esta implica el establecimiento de una relación cognitiva nueva entre dos elementos contextuales. Un niño puede manifestar originalidad, a escala intrapersonal e interpersonal, debido a su desconocimiento de los patrones culturales, de lo ya hecho, y porque no está viciado con los paradigmas cotidianos utilizados para reflejar la realidad por el hombre educado en esa sociedad (Mongeotti, 2001). El bajo nivel de originalidad junto con flexibilidad y organización registrados aquí podría significar, entonces, que la educación está en camino de parametrar a los niños en la manera de enfrentar y solucionar problemas. Es interesante recordar que Guencer y Oral (1993) encontraron que la creatividad se relaciona inversamente con la aceptación de la disciplina en la escuela y que los niños en estudio están en proceso de interiorizar los modelos dominantes en la sociedad. Ello pondría de relieve que la educación formal e informal que reciben los niños, aunque no favorece la flexibilidad, la originalidad ni la organización, por lo menos parece no haber tenido el mismo papel en lo concerniente a la fluidez, es decir en la cantidad de respuestas que el individuo es capaz de dar frente a una situación. No obstante, la cantidad de respuestas sin flexibilidad, originalidad y organización es simplemente cuantitativa (Ullman, 1972, citado por Sánchez, 2003).

No se puede estar seguros de que estos efectos de la educación en la creatividad del niño hayan sido previstos por los especialistas; aunque es mucho más probable que no. No obstante, los resultados que aquí se discuten pueden ser de mucha importancia para los responsables de la educación en el Perú. Por lo menos en lo que a la educación formal se refiere. Cabe mencionar que la creatividad no es una cualidad innata en el ser humano, sino que se desarrolla en virtud de la estimulación que este recibe y es por tanto una tarea del sistema educativo lograr su desarrollo. 


\section{CONCLuSIONES}

La variable estado nutricional ha mostrado ser poco distintiva en lo que a la creatividad se refiere. Los resultados conducen a pensar que los niños pueden ser más o menos creativos en general, independientemente de su estado nutricional, por lo menos respecto del déficit nutricional crónico expresado en menor talla para la edad. Se ha ratificado con ello la tesis según la cual la forma más elevada del psiquismo sería más permeable a la influencia de los factores ambientales que del estado nutricional

Por otro lado, se ha podido observar que la creatividad, así como cada uno de sus indicadores (fluidez, flexibilidad, organización y originalidad), no siguen un patrón de desarrollo uniforme en función de la edad ni del grado de instrucción. Muy por el contrario, se han revelado oscilaciones, e incluso caídas, en los puntajes del último grado de instrucción y del grupo de mayor edad estudiado. Lo cual hace plausible pensar que el tener mayor edad o mayor grado de instrucción no necesariamente significa ser más creativo y pone en el tapete la propuesta de Torrance, quien plantea que hay etapas en que la educación pone más frenos al desarrollo de la creatividad.

Los resultados han revelado también que sí se hallan algunas diferencias al comparar varones con mujeres, ambos con déficit nutricional. Esto conduce a pensar que probablemente los factores asociados a la educación de uno y otro sexo, en una sociedad machista, se hallen en la base de tales diferencias; dotando a los varones de mayor fluidez en sus respuestas y a las mujeres de mayor capacidad de organización.

Un aspecto no poco relevante es el de haber encontrado que ambos grupos (con déficit nutricional y sin él) se ubican en el nivel medio o dentro de lo esperado en creatividad en general, así como en fluidez. Pero lo más notable es que se hallan por debajo de la norma en flexibilidad, originalidad y organización. Todo ello ha conducido a pensar que la educación formal e informal no está ejerciendo el mismo efecto sobre la creatividad y sus componentes. Y que algunos de ellos son menos favorecidos que otros.

Finalmente, el trabajo ha permitido contribuir a la validación y confiabilidad de una prueba de creatividad, que en condiciones corrientes puede ser aplicada en forma colectiva a niños de ocho a 12 años. Y en forma individual a niños de siete. Asimismo, se han elaborado los primeros parámetros válidos por lo menos para los estudiantes de los dos últimos ciclos de educación regular escolarizada y diurna de estatus socioeconómico medio y bajo de Lima metropolitana. 


\section{REFERENCIAS}

Burgos, G. (1999). La creatividad de los estudiantes de sexto grado en las escuelas públicas de Puerto Rico. Teseo [en línea] recoge y permite recuperar información acerca de las tesis doctorales presentadas en las universidades españolas desde 1976 <http:// www.mcu.es/TESEO $>$ [Consulta: 16 de mayo de 1999].

Deusa, F. (1999). "Creatividad escolar y medio socioeconómico y cultural". Curso: 9091. Teseo [en línea] <http:// www.mcu.es/TESEO $>$ [Consulta: 16 de mayo de 1999].

Espriu, R. (1993). El niño y la creatividad. México: Trillas.

Garaigordobil, M. \& Torres, E. (1997). Evaluación de la creatividad en sus correlatos con inteligencia y rendimiento académico. Tarraconenses, 18 (1), 87-98. Universidad del País Vasco.

García Jiménez, M. V. \& Yela, M. (1994). Estudio de los procesos que subyacen a las dimensiones de la fluidez verbal. Afiliación de la Universidad Complutense de Madrid. Revista de Psicología General y Aplicada, 47 (3), 267-273.

Guencer, B. \& Oral, G. (1993). Relación entre creatividad y disconformidad con la disciplina escolar, tal como es percibida por los profesores de estudiantes de escuelas elementales turcas, controlando su grado y sexo (CD-rom). Middle East Technical U, Guenseli Journal of Instructional Psychology, 20 (3), 208-214, abstract del Silver Platter Archivo PsycLIT. Registro ID: 199415508-001.
Hoffman, L.; París, S. \& Hall, E. (1996). Psicología del desarrollo hoy. Madrid: Mc.Graw-Hill.

Instituto Nacional de Investigación y Desarrollo Educativo (1986). Encuesta Nacional de Nutrición y Salud de 1984. Lima: Inide.

Kallin, D. (1973) Nutrición, desarrollo y comportamiento social. Revista de la $O P S$, publicada en la serie Publicación Científica (EE UU) 269.

Kattami, H.; Elkaissi, H. \& Kattami, Y. (1995). La relación entre creatividad y aprovechamiento escolar y otras variables sociales, económicas y culturales en estudiantes de décimo grado de la ciudad de Ahman. Dirasat, febrero, 22. ${ }^{\mathrm{a}}$ (1), 217-251, Abstract del Silver Platter Archivo PsycLIT. Registro ID: 199786930-001

Khanam, M. \& Sen, A. (1998). La influencia de la creatividad, el sexo y el tipo de colegio en la autopercepción creativa. Social Science International, Jan 1998, 14 (1-2), 60, abstract del Silver Platter Archivo PsycLIT. Registro ID: 199738892-007.

Kim, J. \& Michael, W. (1995). La relación de las medidas de creatividad con el aprovechamiento escolar y el estilo de pensamiento y de aprendizaje preferidos en una muestra de estudiantes coreanos de high school. International Journal of Psychology, 30 (4), 417-430, abstract del Silver Platter Archivo PsycLIT. Registro ID: 1996-21620-001.

Klein, R. (1983). Factores nutricionales y socioeconómicos en el desarrollo mental y rendimiento escolar. Revista de la Sociedad Venezolana de Gastroenterología, GEN. 37 (1-2), 167-198. Caracas. 
Lui-Lam, M. (1998). Inteligencia, creatividad y ajuste psicosocial en niños talentosos. Tesis para optar el título profesional. Lima: Universidad Ricardo Palma.

Matalinares, M. (1989). Efectos de la desnutrición en el desarrollo de la atención en niños en edad escolar. Tesis de licenciatura. Lima: Universidad Ricardo Palma.

Matalinares, M. \& Lazarte, C. (2002). El desarrollo de la creatividad en escolares de Lima metropolitana. Revista de Post Grado de la Facultad de Psicología. VII, 1, 17-30.

Mayer, R. (1986). Pensamiento, resolución de problemas y cognición. Barcelona: Paidós.

Merril, R. \& Felson, D. (1976). Desnutrición, aprendizaje y comportamiento. Serie Publicación Científica, auspiciada por la OPS, 352, 1-43.

Mongeotti, P. (2001). La creatividad. Hacia un modelo psicológico explicativo. Revista Cubana de Psicología, 18 (3), 235-244.

Moreno, J. (1992). La capacidad creadora y los aprendizajes escolares. Estudio de los factores constitutivos de la creatividad. Revista de Psicología de la Educación de la Universidad de Castilla-LaMancha, 3 (9), 15-26.

Napoli, R. \& Horton, E. (1997). Necesidades energéticas. Artículo de la publicación científica Conocimientos actuales sobre nutrición, 565, Washington: OPS-OMS.

Ocrospoma, R. (1984). Efectos de la desnutrición en las habilidades básicas para el aprendizaje. Tesis para optar el título profesional. Lima: Universidad Nacional Mayor de San Marcos.
Ocrospoma, R., Yarlequé, L., Matalinares, M. y Fernández Baca, D. (1991). Efectos de la desnutrición en la psicomotricidad, atención y memoria de los niños en edad escolar de zonas urbanomarginales. Lima: Concytec.

Oerter, R. (1975). Psicología del pensamiento. Barcelona: Herder.

Olaseinde, O. (1994). La relación de la dimensión del estilo cognitivo de impulsividad/reflexividad con la creatividad en estudiantes secundarios. IFE Psychologia: An International Journal, 2 (1), 64-74.

Ortega, M. (1999). La orientación de la creatividad en el niño preescolar. Curso: 9091. Teseo [en línea]. La Secretaría del Consejo de Universidades <http:/www.mcu.es/ TESEO $>$ [Consulta: 20 de julio de 1999].

Programa de Alimentación y Nutrición para Familiares de Alto Riesgo (Panfar) (1997). Estado nutricional de la niñez en el Perú. Lima: Ministerio de Salud, Programa de Alimentación y Nutrición.

Pollit, E. (1984). La nutrición y el rendimiento escolar. París: OMS (reproducción).

Pollit, E. (2002). Consecuencias de la desnutrición en el escolar peruano. Lima: Pontificia Universidad Católica del Perú, Fondo Editorial.

Sánchez, H. (1991). Prueba para evaluar indicadores básicos de creatividad (EIBC). Revista de Psicología de la Universidad Ricardo Palma, II (1), 28-37.

Sánchez, H. (1995). Inteligencia y creatividad en el niño. Temas de Psicopedagogía 2. Lima: Universidad Ricardo Palma. 
Sánchez, H. \& Reyes, C. (2002). Metodología y diseños en la investigación cientifica. Lima: Universidad Ricardo Palma.

Sánchez, H. (2003). Psicología de la creatividad. Lima: Universidad Ricardo Palma.

Sfeir, R. \& Aguayo, M. (2000). Desnutrición en niños menores de 5 años. Revista del Instituto Médico Sucre, LXV (116), 43-53.

Torres, M. (1996). Implicancias de la nutrición en el desarrollo psicológico de los niños de sectores populares. Tesis para optar el grado de magíster en Psicología. Lima: Universidad Nacional Mayor de San Marcos.

Unicef (1997). Encuesta demográfica y de salud familiar 1996. Lima: Unicef.

Unicef (1997). Estado de la niñez, la adolescencia y la mujer en el Perú 1997. Lima: Unicef.

Unicef (1994). I Censo nacional de talla en escolares 1993. Lima: Unicef/Ministerio de Educación.

Valenciano, J. (1999). Imagen y entrenamiento de la creatividad en expresión escrita. Curso: 9394. Teseo [en línea]. La Secretaría del Consejo de Universidades http://www.mcu.es/ TESEO [Consulta: 20 de julio de 1999].

Yarlequé, L. (1989). Efectos de la desnutrición en el desarrollo de la memoria de niños que cursan educación primaria. Tesis para optar el título profesional. Lima: Universidad Ricardo Palma.

Zavala, M. (1989). Psicomotricidad en niños desnutridos de grado I y II. Tesis para optar el grado de bachiller. Lima: Universidad Ricardo Palma. 